\title{
Numerical Study of Natural Convection Heat Loss from Cylindrical Solar Cavity Receivers
}

\author{
M. Prakash \\ 7 Trinity Enclave, Old Madras Road, Bangalore 560093, India \\ Correspondence should be addressed to M. Prakash; prakashmarath@gmail.com
}

Received 12 November 2013; Accepted 16 December 2013; Published 23 January 2014

Academic Editors: T.-H. Meen and V. Strezov

Copyright (C) 2014 M. Prakash. This is an open access article distributed under the Creative Commons Attribution License, which permits unrestricted use, distribution, and reproduction in any medium, provided the original work is properly cited.

\begin{abstract}
The numerical study of the natural convection loss occurring from cylindrical solar cavity receivers is reported in this communication. These cavity receivers can be used with solar dish concentrators for process heat applications at medium temperature levels. Three cylindrical cavity receivers of diameter $0.2,0.3$, and $0.4 \mathrm{~m}$ with aspect ratio equal to one and opening ratios of 1 and 0.5 are used for the analysis. Fluent CFD software is used for the analysis of the three-dimensional (3D) receiver models. In this study the receiver tubes within the cylindrical cavity are modeled as a helical coil similar to those existing in actual systems. The flow of the working fluid within the helical coil is also modeled. The simulations are performed for fluid inlet temperatures of $150^{\circ} \mathrm{C}$ and $250^{\circ} \mathrm{C}$ and for receiver inclination angles of 0 (sideways-facing cavity), 30, 45, 60, and 90 degree (vertically downwardfacing receiver). It is found that the convective loss increases with increasing mean fluid temperature and decreases with, increase in receiver inclination. The convective loss is found to increase with, opening ratio. These observations are true for all cavity receivers analysed here. A Nusselt number correlation involving Rayleigh numbers, receiver inclinations, and opening ratios is proposed for the convective loss.
\end{abstract}

\section{Introduction}

Cavity receivers are widely used along with solar dish systems for providing industrial process heat $[1,2]$ generating electric power $[3,4]$ and for thermochemical reactions [5]. The overall efficiency of such systems is dependent on the thermal losses occurring from the cavity receiver. It is observed from the literature that the convective heat transfer constitutes a major share of the thermal losses [6-8]. The convective losses from these solar cavity receivers are found to be dependent on various parameters like receiver inclination $(\theta)$, receiver wall boundary condition, aspect ratio $(L / D)$, opening ratio $(d / D)$, and external wind. This is the reason due to which analysis of convective losses from solar cavity receivers is complicated when compared to that of heat transfer due to radiation and conduction $[9,10]$.

Experimental and numerical investigations on natural convection losses in cavity receivers used with solar dish concentrators have been performed on different cavity shapes.
Stine and McDonald [9] have performed experimental studies on conical frustum-cylindrical cavity receivers while Kugath et al. [1] has performed field studies on a similar receiver shape at medium temperature levels. Cylindricalshaped cavity receivers have been analysed experimentally and numerically $[6,8,9,11,12]$. Numerical investigations on hemispherical cavity receivers have been carried out by Sendhil Kumar and Reddy $[7,13]$. The effect of wall temperatures, receiver inclination, and $d / D$ ratio on the natural convection loss has been analysed. Nusselt number correlations have been proposed.

It is observed from the literature that the majority of the numerical studies on natural convection loss are performed on cavity receivers used for high temperature applications $\left(>400^{\circ} \mathrm{C}\right)$ such as power generation. The cavity receivers used with solar dish systems for process heat applications are generally cylindrical in shape, operate at medium temperatures (below $300^{\circ} \mathrm{C}$ ), have $d / D$ ratios even greater than one, and may have nonuniform wall temperatures. The receiver 


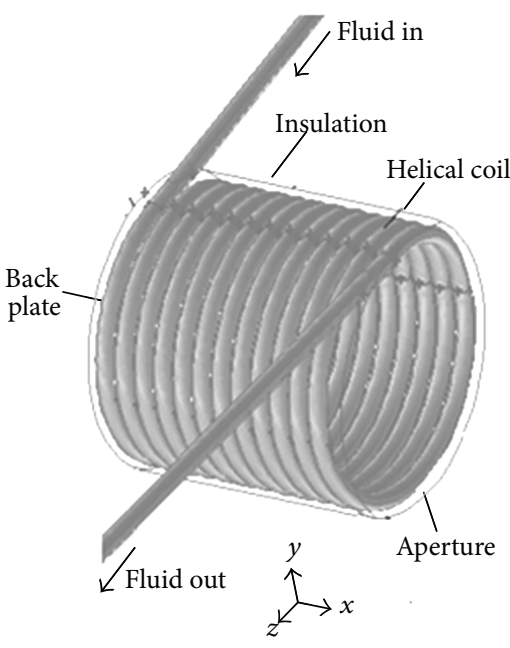

(a) Cavity receiver model

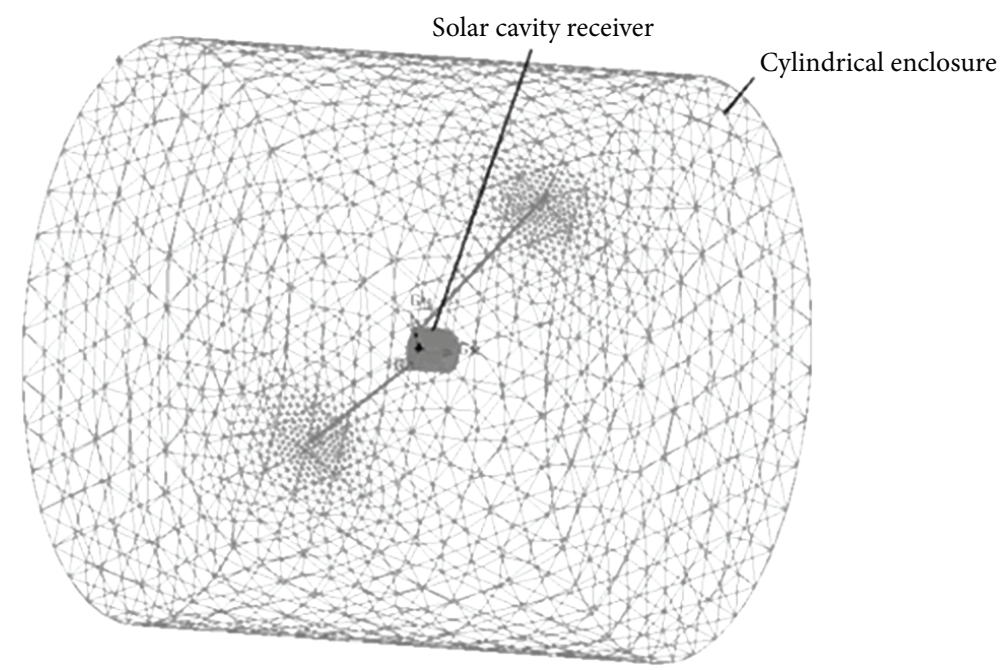

(b) The mesh used for simulation

FIGURE 1: Cavity receiver model and the mesh used for the simulation.

used for the process heat applications consists of a receiver tube assembly enclosed within a cylindrical open cavity. This particular design of the receiver does not have stringent constraints on tube volume and pressure drop and is easy to fabricate making it different from those used for high temperature applications.

Solar cavity receivers used for high temperature applications are generally modeled as plain walls with isothermal boundary conditions and have $d / D$ ratios equal to or less than one $[7,14]$. Numerical modeling of the cylindrical cavity receivers encountered in process heat applications (temperatures below $300^{\circ} \mathrm{C}$ ) is limited. The numerical studies performed by Prakash et al. $[8,12]$ have been carried out on such a cavity receiver. The use of Fluent CFD software for modeling the receiver tubes and the flow within the tubes has been demonstrated by these studies for the cavity receiver having $d / D$ ratio greater than one. It is therefore noticed that numerical study of natural convection loss from cylindrical cavity receivers of various diameters used for process heat applications at temperature levels of about $150^{\circ} \mathrm{C}-250^{\circ} \mathrm{C}$ having $d / D$ ratio of 1 and 0.5 and inclined at different angles has not been performed.

In this present communication, numerical analysis of the natural convection loss occurring from three cylindrical cavity receivers of diameter $0.2,0.3$, and $0.4 \mathrm{~m}$ with $L / D$ equal to one and $d / D$ ratios equal to 1 and 0.5 is reported. The receiver tubes are modeled as a helical coil and the flow of the working fluid within the helical coil is also modeled. The simulations are performed for fluid inlet temperatures of $150^{\circ} \mathrm{C}$ and $250^{\circ} \mathrm{C}$ and for receiver inclination angles of 0 (side-ways-facing cavity), 30, 45, 60, and 90 degree (vertically downward facing-receiver).

\section{Numerical Model and Procedure}

Three cavity receivers of diameter $0.2,0.3$, and $0.4 \mathrm{~m}$ are used for the analysis. Each receiver is modeled as a helical coil having coil external diameter equal to the cavity receiver diameter $(D)$ using the Gambit tool of Fluent CFD software package [15]. The helical coil has multiple turns such that the length of the cavity is equal to the cavity diameter $(L / D=1)$. The receiver aperture diameter $(d)$ is equal to the cavity receiver diameter $(D)$ for opening ratio of 1 and is half the cavity receiver diameter for opening ratio of 0.5 . The helical coils for the $0.3 \mathrm{~m}$ and $0.4 \mathrm{~m}$ diameter receiver are modeled using a metallic tube having $0.025 \mathrm{~m}$ diameter while a metallic tube of $0.0125 \mathrm{~m}$ is used to model the receiver of $0.2 \mathrm{~m}$ diameter. The pitch between the coil turns is about $0.004-0.005 \mathrm{~m}$. The helical coils are surrounded on all sides except the aperture by a layer of insulation giving the receiver assembly a cylindrical shape. The model for the $0.4 \mathrm{~m}$ diameter cavity receiver (opening ratio of 1 ) along with the insulation is shown in Figure 1(a).

The cavity receiver along with the insulation is surrounded by a cylindrical enclosure. This is done as the boundary condition at the aperture is unknown and also to ensure that the air flow within the cavity is unaffected by the conditions external to the receiver. It is found from a preliminary analysis that an enclosure size about ten times the cavity receiver diameter affects the air flow within the cavity receiver while an enclosure about twenty times the cavity diameter takes higher computational time. Therefore, an enclosure size of about fifteen times the cavity receiver diameter is found to be suitable for the analysis because the air flow within cavity is unaffected by the enclosure and the computational time is also reduced. The fluid inlet and the outlet tubes of the receiver are extended to the enclosure walls and are assumed to be adiabatic so that they do not affect the temperature and flow profile in the region external to the cavity. The fluid flow in the model is reverse to the actual flow within the receiver operating in the field. This is similar to the approach used in the experiments $[8,9$, 16]. This is done to ensure that the back plate region has the highest fluid temperature value when compared to the 
aperture region as observed in cavity receivers operating in field. The modeling of fluid flow within the helical coil also leads to non-uniform receiver wall temperatures similar to those observed in experiments [9] and field studies [2].

A fine mesh is used within the metallic tube of the helical coil (interval size of about 4-10) and the region between the helical coil and the insulation (interval size of about 15-20). A coarse mesh is used for the enclosure (interval size of about 500). The mesh coarsens progressively as the enclosure walls are approached from the centre of the cavity. The mesh used for the simulation of the receiver is shown in Figure 1(b). Copper is selected as the material of the receiver tubes while DOWTHERM A [17] is selected as the fluid flowing within the receiver tubes (also referred to as the working fluid). The interior of the cavity and the enclosure is filled with air. The material properties of copper and air are taken from Kothandaraman and Subramanyan [18]. The Boussinesq approximation is used for the air properties.

The boundary conditions used for the numerical analysis are as follows:

(1) the inlet temperature and the velocity of the working fluid are specified,

(2) adiabatic condition is assumed for the cavity insulation;

(3) cylindrical enclosure walls are maintained at ambient temperature of $30^{\circ} \mathrm{C}$.

The fluid inlet temperatures are $150^{\circ} \mathrm{C}$, and $250^{\circ} \mathrm{C}$. The fluid velocities are 0.21 , and $0.235 \mathrm{~m} / \mathrm{s}$ at $150^{\circ} \mathrm{C}$, and $250^{\circ} \mathrm{C}$ respectively, for the $0.3 \mathrm{~m}$ and $0.4 \mathrm{~m}$ cavity receivers. The fluid velocities are 0.42 and $0.47 \mathrm{~m} / \mathrm{s}$ at $150^{\circ} \mathrm{C}$ and $250^{\circ} \mathrm{C}$ respectively, for the $0.2 \mathrm{~m}$ cavity receiver. This is to ensure that at each fluid inlet temperature, the Reynolds number within the helical coil for all the receiver sizes is the same. The $\mathrm{k}-$ $\varepsilon$ turbulence model is used for the analysis as the Reynolds number of the working fluid within the helical coil indicates turbulent flow (Srinivasan et al. [19]).

The solutions are obtained by solving the continuity equation, the momentum equation, and the energy equation simultaneously. The semi-implicit pressure-linked equation (SIMPLE) scheme of the Fluent software is used. Steady state analysis is carried out. Radiation and conduction heat transfer modes are not analysed. The analysis is performed for different receiver inclinations by adjusting the gravity vector. The momentum and energy solution controls are of the firstorder upwind type so as to reduce the convergence time. The convergence criteria for the residuals of continuity and the velocity equations are of the order of $10^{-4}$ while for the energy equation, $10^{-7}$. The solutions are obtained once the convergence criteria are satisfied.

\section{Results and Discussions}

The steady state outlet temperature and mass flow rate of the working fluid are obtained from each numerical simulation performed in this study. The temperature drop of the working fluid within the helical coil is taken as the convective loss occurring from the receiver. The natural convection loss is then calculated as

$$
Q_{\text {conv }}=\dot{m} c_{p}\left(T_{f, \text { in }}-T_{f, \text { out }}\right),
$$

where $\dot{m}$ is the fluid mass flow rate, $c_{p}$ is the specific heat of the working fluid, $T_{f \text {,in }}$ is the fluid inlet temperature, $T_{f \text {,out }}$ is fluid outlet temperature, and $T_{a}$ is the ambient temperature.

The convective loss values at different fluid inlet temperatures are plotted against inclination for the cavity receiver with opening ratio of 1 . This is shown in Figure 2. It can be seen that the convective losses increase with increasing fluid inlet temperature and decrease with receiver inclination. The mass flow rate of the working fluid is kept constant (mass flow rate of $0.088 \mathrm{~kg} / \mathrm{s}$ for 0.3 and $0.4 \mathrm{~m}$ diameter receivers and $0.045 \mathrm{~kg} / \mathrm{s}$ for $0.2 \mathrm{~m}$ diameter receiver); hence, higher fluid inlet temperature leads to higher mean fluid temperatures, $T_{m}\left(T_{m}=0.5\left(T_{f \text {,in }}+T_{f \text {,out }}\right)\right.$. The mean fluid temperature is taken as the mean receiver temperature and an increase in this temperature leads to higher convective currents of air within the cavity causing higher convective losses.

The decrease in the convective loss with the increase in receiver inclination is due to the formation of stagnation zone of air within the cavity as the cavity is tilted from $0^{\circ}$ to $90^{\circ}$. The higher the stagnation zone area, the lower the convective losses. The stagnation zone area increases with inclination due to which the convective loss decreases as reported in the literature $[12,20]$. It is observed that the convective loss values decrease by about $80 \%$ when the receiver inclination is increased from $0^{\circ}$ to $90^{\circ}$. The loss values also increase with receiver size due to an increase in the receiver tube area (heat transfer area). Similar trends are observed when the convective loss values for the cavity receivers with opening ratio of 0.5 are plotted against inclination. This is shown in Figure 3.

Figure 4 shows the comparison of the convective loss values for cavity receivers with opening ratio of 1 and 0.5 for fluid inlet temperature of $150^{\circ} \mathrm{C}$. It can be observed that the convective loss values increase with opening ratio. The higher the opening ratio, the greater the convective zone leading to higher convective losses. An increase of about $30-50 \%$ in the convective loss values is observed when the opening ratio increases from 0.5 to 1 . The trend is similar to fluid inlet temperature of $250^{\circ} \mathrm{C}$.

The convective loss values obtained from the numerical simulations are used to develop Nusselt number correlation. The Nusselt number based on the cavity diameter $(D)$ for the natural convection heat transfer from the receiver tube surface to the air within the cavity is given by

$$
\mathrm{Nu}_{D}=\frac{h D}{k}
$$

where $h$ is the convective heat transfer coefficient between the receiver tube surface and the air within the cavity, $D$ is the cavity diameter, and $k$ is the thermal conductivity of air. The air properties are taken at the film temperature which is the 


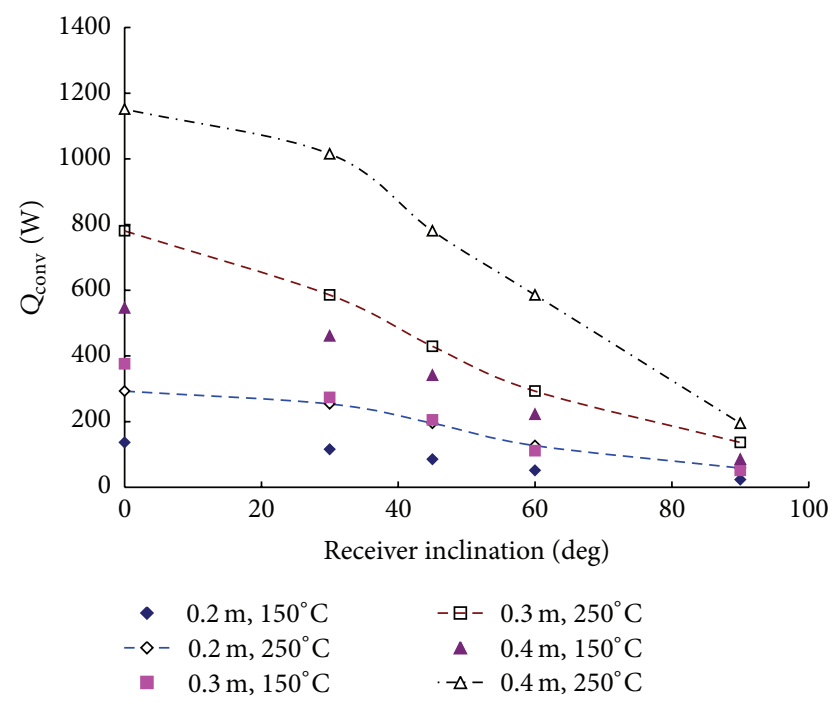

FIGURE 2: Variation of the natural convection loss with inclination for different fluid inlet temperatures and receiver diameters with opening ratio $=1$.

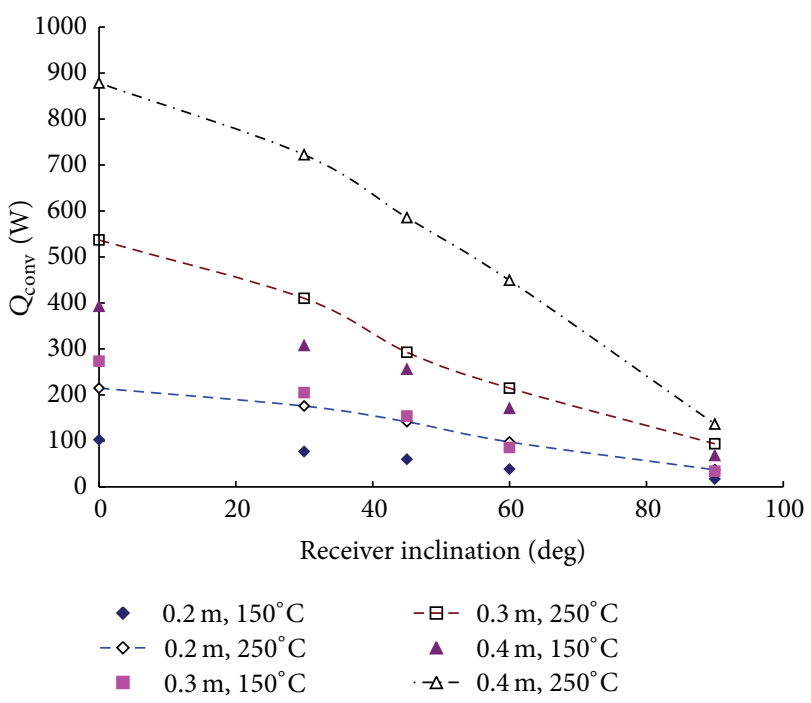

FIGURE 3: Variation of the natural convection loss with inclination for different fluid inlet temperatures and receiver diameters with opening ratio $=0.5$.

average of $T_{m}$ and $T_{a}$. The convective heat transfer coefficient $(h)$ is given by

$$
h=\frac{Q_{\text {conv }}}{A_{w}\left(T_{m}-T_{a}\right)},
$$

where $A_{w}$ is the total receiver tube area, $T_{m}$ is the mean fluid temperature, and $T_{a}$ is the ambient temperature.

A correlation is proposed involving the Nusselt numbers, Rayleigh numbers, the receiver inclination values and opening ratio values. The Rayleigh numbers are calculated based

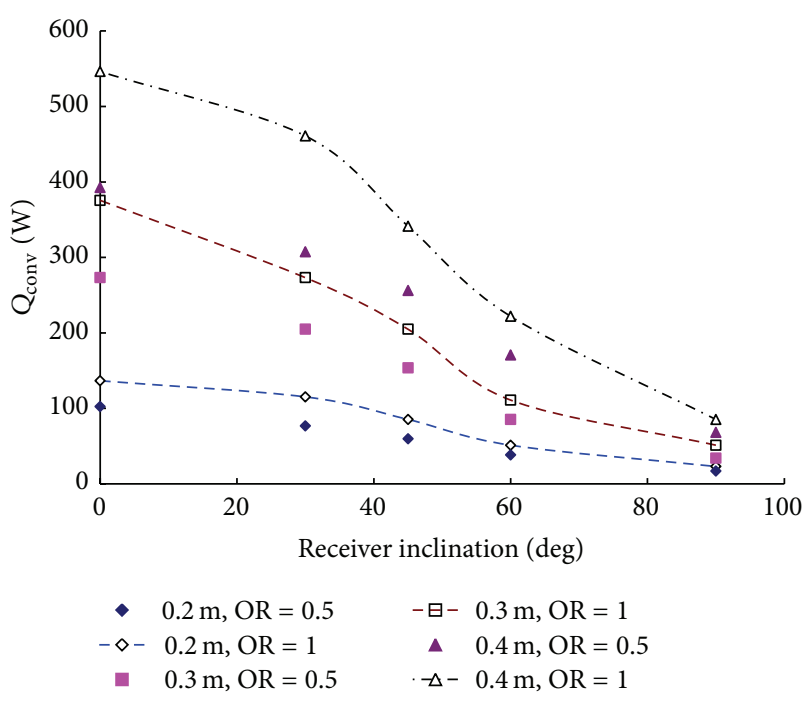

FIgURE 4: Comparison of the convective loss values for cavity receivers with opening ratio of 1 and 0.5 for fluid inlet temperature of $150^{\circ} \mathrm{C}$.

on the receiver diameter. The Nusselt number correlation is given by

$$
\mathrm{Nu}_{D}=0.0133 \mathrm{Ra}_{D}{ }^{(1 / 3)}(1+\cos \theta)^{2.6}\left(\frac{d}{D}\right)^{0.47} .
$$

The above correlation is proposed for $\mathrm{Ra}_{D}$ between $3.7 \times$ $10^{7}$ and $3.1 \times 10^{8}$ and for $T_{m}$ between $148^{\circ} \mathrm{C}$ and $250^{\circ} \mathrm{C}$. Figure 5 shows the parity plot between the Nusselt numbers obtained from the numerical simulations and the proposed correlation. A good agreement is noticed with $90 \%$ of the data within the $\pm 10 \%$ variation band.

The applicability of the proposed correlation is checked for cylindrical cavity receivers operating at higher temperatures and having dimensions other than those analysed here. The correlation is applied to the model cavity receiver described by Taumoefolau et al. [6]. The comparison of the natural convection loss obtained from the experiments [6] and the proposed correlation at temperature of $550^{\circ} \mathrm{C}(823 \mathrm{~K})$ is shown in Figure 6. A fairly good agreement is observed between the experimental and correlation values with maximum deviation of about $30 \%$. The average deviation is about $15 \%$. A similar deviation is noticed when the values are compared for $450^{\circ} \mathrm{C}$. A maximum variation of about $30 \%$ is observed when the convection heat flux predicted by the present correlation is compared with those reported for the model receiver with an aspect ratio of 1.1 [14]. The deviations observed from the comparisons may be due to the reason that the receiver analysed in the present work is modeled as a helical coil while the experiments performed by Taumoefolau et al. [6] are on a cylindrical receiver with plain walls. The present correlation is also developed for a range of Rayleigh numbers between $3.7 \times 10^{7}$ and $3.1 \times 10^{8}$ while the Rayleigh numbers in the study by Taumoefolau et al. [6] are of the order of $10^{6}$. 


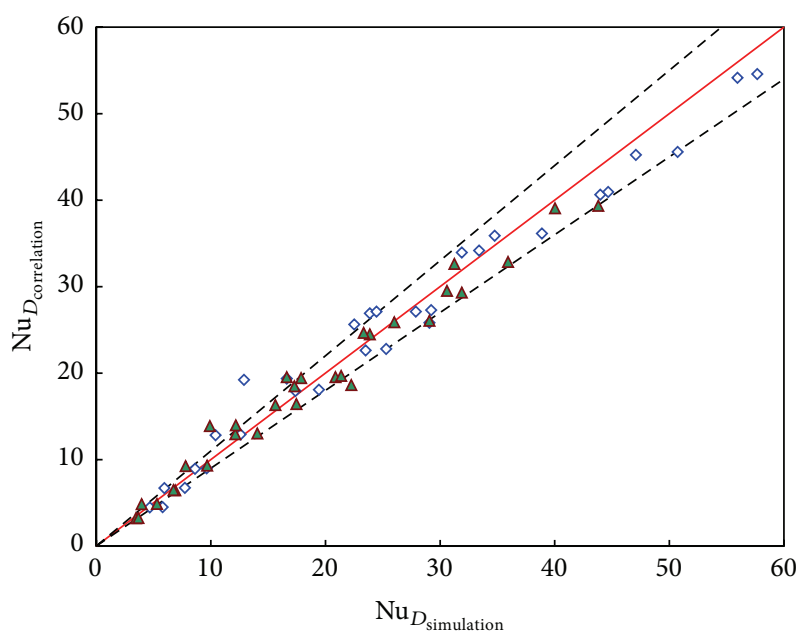

$\diamond \mathrm{OR}=1$

$\triangle \mathrm{OR}=0.5$

FIGURE 5: Parity plot between $\mathrm{Nu}_{D_{\text {simulation }}}$ and $\mathrm{Nu}_{D_{\text {correlation }}}$.

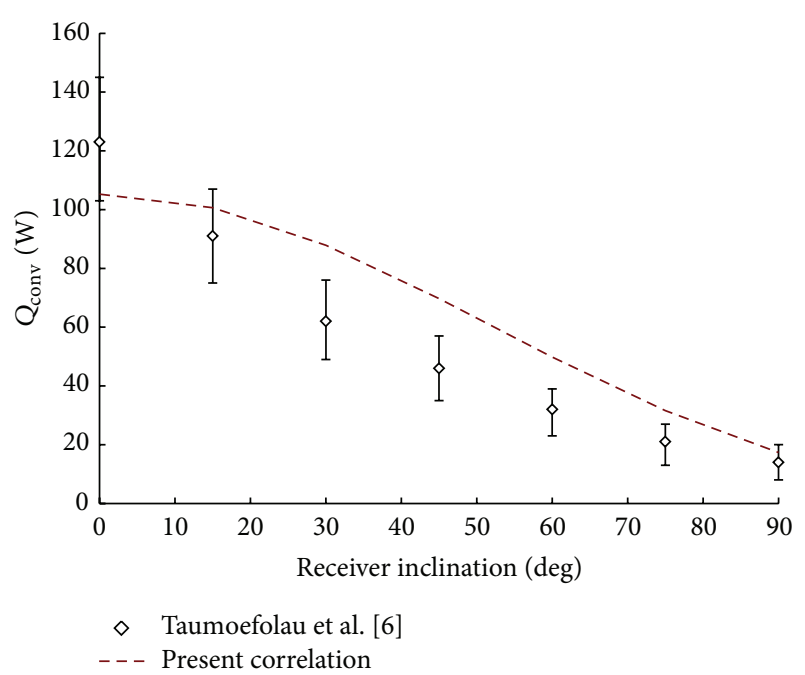

FIGURE 6: Comparison of the natural convection loss obtained from the experiments (Taumoefolau et al. [6]) and present correlation at temperature of $550^{\circ} \mathrm{C}(823 \mathrm{~K})$.

The present correlation is applied to the cylindrical cavity receiver studied by Lezhebokov et al. [21]. The convective loss values obtained for $30^{\circ}$ and $60^{\circ}$ inclination at $500^{\circ} \mathrm{C}$ and $600^{\circ} \mathrm{C}$ receiver temperatures are compared. It is found that the maximum variation between the convective loss values reported by Lezhebokov et al. [21] and the present correlation is about $25 \%$. The present correlation can therefore be used to determine the natural convection loss at receiver inclinations between $0^{\circ}$ and $90^{\circ}$ in an approximate manner from cylindrical cavity receivers operating at higher temperatures (about $550^{\circ} \mathrm{C}$ ) having $d / D$ ratio of 1 . Prakash et al. $[8,12]$ performed studies on a cylindrical cavity receiver having $d / D$ ratio greater than one; hence, a comparison with the results from those studies is not attempted.

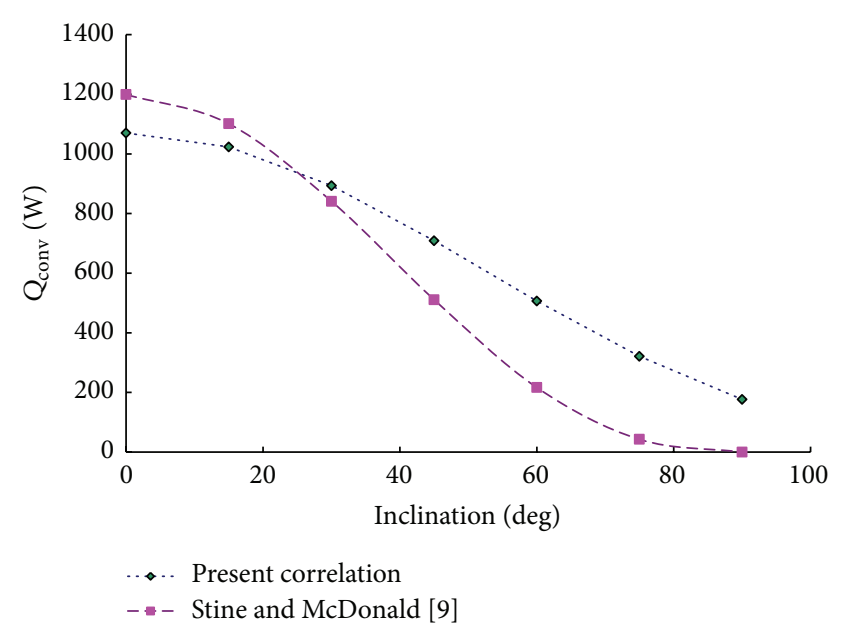

Figure 7: Comparison of the natural convection loss obtained from the experiments (Stine and McDonald [9]) and present correlation at temperature of $205^{\circ} \mathrm{C}$, opening ratio of 0.69 .

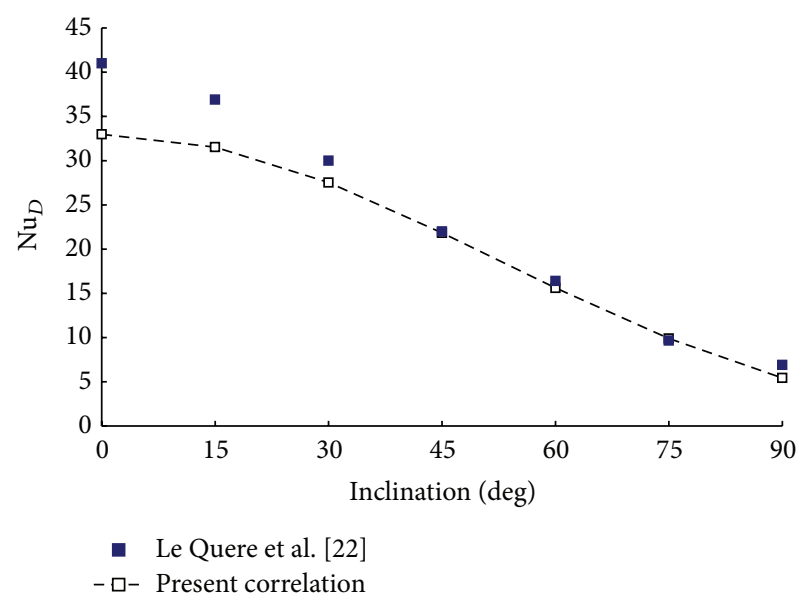

FIGURE 8: Comparison of Nusselt numbers obtained from experiments (Le Quere et al. [22]) and from present correlation at Grashof number of $1 \times 10^{8}$.

The present correlation is applied to the conical frustumcylindrical cavity receiver described by Stine and McDonald [9] having opening ratio of 1 and 0.69 . The natural convection loss values predicted for the receiver by the present correlation are compared with those obtained from the experiments carried out by Stine and McDonald [9] for $205^{\circ} \mathrm{C}$. Figure 7 shows the comparison of the convective losses obtained experimentally and those predicted by the present correlation for the conical frustum-cylindrical cavity receiver with opening ratio of 0.69 . It is observed that the agreement between the values is good (maximum variation of about $30 \%$ ) for inclinations between $0^{\circ}$ and $45^{\circ}$ while the variations are very high for the $60^{\circ}$ and $75^{\circ}$ inclinations. A similar trend is observed for the receiver with opening ratio of 1 operating at $205^{\circ} \mathrm{C}$. The variations in the convective loss values may be due to the fact that the shape of the receiver analysed by Stine and McDonald [9] and McDonald [16] is 
different from the receiver of the present study. The Rayleigh numbers observed from the experiments are of the order of $1.5 \times 10^{9}$ while the present correlation has been developed for Rayleigh numbers of about $3 \times 10^{8}$. It is also noticed that the natural convection loss values reported in the studies by Stine and McDonald [9] and McDonald [16] are limited to inclinations between $0^{\circ}$ and $75^{\circ}$ and the convection loss at $90^{\circ}$ is neglected whereas the convective loss values at $90^{\circ}$ are also considered for developing the new correlation. The correlation developed from the present study has a fairly good agreement with the Stine and McDonald [9] experimental values for receiver inclinations between $0^{\circ}$ and $45^{\circ}$.

The applicability of the correlation developed in this study for prediction of natural convection loss in cubical cavity receivers is checked. The study by Le Quere et al. [22] involving cubical cavity receivers is used for the comparison. This study [22] is selected as it involves Rayleigh numbers of the order similar to that of the present study. Figure 8 shows the experimental (Le Quere et al. [22]) and predicted Nusselt numbers obtained for a downward facing cubical open cavity of side $0.6 \mathrm{~m}$ at a Grashof number of about $1 \times 10^{8}$ (Rayleigh number of $\left.7 \times 10^{7}\right)$. The maximum variation is of the order of $20 \%$. The experimental receiver of Le Quere et al. [22] is cubical in shape and is made of plain walls unlike the cylindrical receiver analysed in the present study involving receiver tubes. The variation in the loss values may be due to this fact and also due to experimental uncertainties. It can be therefore observed that the correlation can be used with fair accuracy for predicting the natural convection loss from a downward facing cubical cavity receiver with opening ratio of 1 and Rayleigh number of the order of $10^{8}$.

\section{Conclusions}

In this communication, the natural convection loss occurring from downward facing cylindrical cavity receivers used with solar dish concentrators for medium temperature applications is studied numerically. The effects of fluid inlet temperature, receiver inclination, and opening ratio on the convective losses are analysed. The following conclusions are drawn from this study.

(1) The convective losses increase with an increase in mean fluid temperature for all receiver sizes and inclinations.

(2) The convective losses decrease with an increase in receiver inclination. It is observed that the convective loss values decrease by about $80 \%$ when the receiver inclination is increased from $0^{\circ}$ to $90^{\circ}$. The variation of the convective losses with inclination is non linear.

(3) A new correlation is proposed involving Nusselt number, Rayleigh number, the receiver inclination, and opening ratio for cylindrical cavity receivers operating at medium temperatures $\left(100-300^{\circ} \mathrm{C}\right)$. The correlation is developed with a coefficient of determination $\left(R^{2}\right)$ of 0.97 . This correlation will be useful for determining the natural convection loss occurring from the solar dish-cavity receiver systems used for medium temperature process heat applications.

(4) The present correlation can also be used to determine the natural convection loss at receiver inclinations between $0^{\circ}$ and $90^{\circ}$ in an approximate manner from cylindrical cavity receivers having $d / D$ ratio of 1 operating at higher temperatures (about $550^{\circ} \mathrm{C}$ ). The correlation can also be used with fair accuracy for predicting the natural convection loss from a downward facing cubical cavity receiver with opening ratio of 1 and Rayleigh number of the order of $10^{8}$.

(5) Prediction of natural convection loss from conical frustum-cylindrical cavity receivers with opening ratios of 1 and 0.69 is also possible using the present correlation for receiver inclinations between $0^{\circ}$ and $45^{\circ}$. The operating temperatures are in the medium temperature ranges (below $300^{\circ} \mathrm{C}$ ).

\section{Nomenclature}

$A_{w}: \quad$ Total receiver tube area $\left(\mathrm{m}^{2}\right)$

$c_{p}$ : Specific heat of the working fluid

$(\mathrm{kJ} / \mathrm{kg}-\mathrm{K})$

$D: \quad$ Cavity receiver diameter $(\mathrm{m})$

$d: \quad$ Receiver aperture diameter $(\mathrm{m})$

$h$ : Convective heat transfer coefficient $\left(\mathrm{W} / \mathrm{m}^{2}-\mathrm{K}\right)$

$k$ : Thermal conductivity of air $(\mathrm{W} / \mathrm{m}-\mathrm{K})$

$L: \quad$ Receiver length (m)

$\dot{m}$ : Mass flow rate of the working fluid $(\mathrm{kg} / \mathrm{s})$

$\mathrm{Nu}_{D}:$ Nusselt number based on receiver diameter

$Q_{\text {conv }}$ : Convective loss at receiver angle $(\mathrm{W})$

$\mathrm{Ra}_{D}$ : Rayleigh number based on receiver diameter

$T_{a}: \quad$ Ambient temperature $\left({ }^{\circ} \mathrm{C}\right)$

$T_{f, \text { in }}$ : Temperature of working fluid entering the receiver $\left({ }^{\circ} \mathrm{C}\right)$

$T_{f \text {,out }}$ : Temperature of working fluid exiting the receiver $\left({ }^{\circ} \mathrm{C}\right)$

$T_{m}: \quad$ Mean fluid temperature $\left({ }^{\circ} \mathrm{C}\right)$

$\theta: \quad$ Receiver inclination (degrees).

\section{Conflict of Interests}

The author declares that there is no conflict of interests regarding the publication of this paper.

\section{Acknowledgments}

The author wishes to thank Prof J. K. Nayak and Prof S. B. Kedare, Department of Energy Science and Engineering, Indian Institute of Technology Bombay, for their immense encouragement and help. The computations for this work were performed by the author at the Department of Energy Science and Engineering, Indian Institute of Technology Bombay. 


\section{References}

[1] D. A. Kugath, G. Drenker, and A. A. Koenig, "Design and development of a paraboloidal dish solar collector for intermediate temperature service," in Proceedings of the International Solar Energy Society, Silver Jubilee Congress, vol. 1, pp. 449-453, Atlanta, Ga, USA, 1979.

[2] S. B. Kedare, J. K. Nayak, and A. D. Paranjape, "Development, installation and evaluation of large scale concentrating solar collector for medium temperature industrial thermal applications," Final Report of R\&D Project No. 15/9/2002-ST, Ministry of New and Renewable Energy, Government of India, 2006.

[3] K. Lovegrove, A. Zawadski, and J. Coventy, "Taking the anu big dish to commercialization," in Proceedings of the Solar 2006: Clean Energy?-Can Do! (ANZSES '06), Canberra, Australia, 2006.

[4] F. Nepveu, A. Ferriere, and F. Bataille, "Thermal model of a dish/stirling systems," Solar Energy, vol. 83, no. 1, pp. 81-89, 2009.

[5] A. Steinfeld and R. Palumbo, "Solar thermochemical process technology," Encyclopedia of Physical Science \& Technology, vol. 15, pp. 237-256, 2001.

[6] T. Taumoefolau, S. Paitoonsurikarn, G. Hughes, and K. Lovegrove, "Experimental investigation of natural convection heat loss from a model solar concentrator cavity receiver," Journal of Solar Energy Engineering, vol. 126, no. 2, pp. 801-807, 2004.

[7] N. Sendhil Kumar and K. S. Reddy, "Numerical investigation of natural convection heat loss in modified cavity receiver for fuzzy focal solar dish concentrator," Solar Energy, vol. 81, no. 7, pp. 846-855, 2007.

[8] M. Prakash, S. B. Kedare, and J. K. Nayak, "Investigations on heat losses from a solar cavity receiver," Solar Energy, vol. 83, no. 2, pp. 157-170, 2009.

[9] W. B. Stine and C. G. McDonald, "Cavity receiver convective heat loss," in Proceedings of the International Solar Energy Society Solar World Congress, vol. 2, pp. 1318-1322, Kobe, Japan, 1989.

[10] S. Paitoonsurikarn and K. Lovegrove, "A new correlation for predicting the free convection loss from solar dish concentrating receivers," in Proceedings of the Solar 2006: Clean Energy?Can Do! (ANZSES '06), Canberra, Australia, 2006.

[11] S. Paitoonsurikarn and K. Lovegrove, "Numerical investigation of natural convection loss in cavity-type solar receivers," in Proceedings of the Solar, ANZSES Annual Conference, Newcastle, Australia, 2002.

[12] M. Prakash, S. B. Kedare, and J. K. Nayak, "Determination of stagnation and convective zones in a solar cavity receiver," International Journal of Thermal Sciences, vol. 49, no. 4, pp. 680691, 2010.

[13] N. Sendhil Kumar and K. S. Reddy, "Comparison of receivers for solar dish collector system," Energy Conversion and Management, vol. 49, no. 4, pp. 812-819, 2008.

[14] S. Paitoonsurikarn, K. Lovegrove, G. Hughes, and J. Pye, "Numerical investigation of natural convection loss from cavity receivers in solar dish applications," Journal of Solar Energy Engineering, vol. 133, no. 2, Article ID 021004, 2011.

[15] Fluent CFD software package, version 6. 3. 26, 2006.

[16] C. G. McDonald, "Heat loss from an open cavity," Sandia National Laboratories Report SAND95-2939, 1995.

[17] The Dow Chemical Company, "DOWTHERM A heat transfer fluid," Product Technical Data, 1997.
[18] C. P. Kothandaraman and S. Subramanyan, Heat and Mass Transfer Data Book, New Age International Publishers, New Delhi, India, 4th edition, 1998.

[19] P. S. Srinivasan, S. S. Nandapurkar, and F. A. Holland, "Pressure drop and heat transfer in coils," The Chemical Engineer CE113119, 1968.

[20] A. M. Clausing, "Convective losses from cavity solar receiverscomparisons between analytical predictions and experimental results," Journal of Solar Energy Engineering, vol. 105, no. 1, pp. 29-33, 1983.

[21] A. I. Lezhebokov, Y. B. Sokolova, and V. S. Trukhov, "Heat losses from a receiver of concentrated radiation in a solar energy unit with thermodynamic transducer," Applied Solar Energy, vol. 22, no. 2, pp. 34-38, 1986.

[22] P. Le Quere, F. Penot, and M. Mirenayat, "Experimental study of heat loss through natural convection from an isothermal cubic open cavity," in Proceedings of the DOE/SERI/SNLL Workshop on Convective Losses from Solar Receivers, pp. 165-174, Sandia Laboratory Report, SAND81-8014, 1981. 


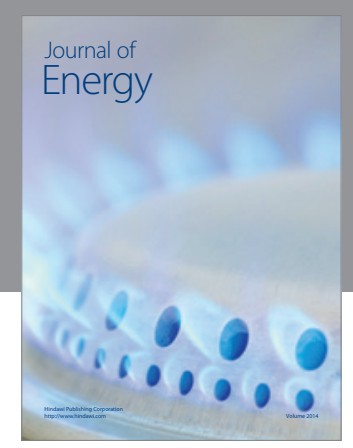

Journal of

Industrial Engineering
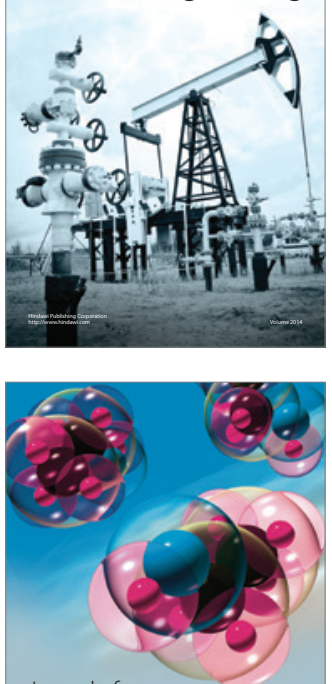

Fuels
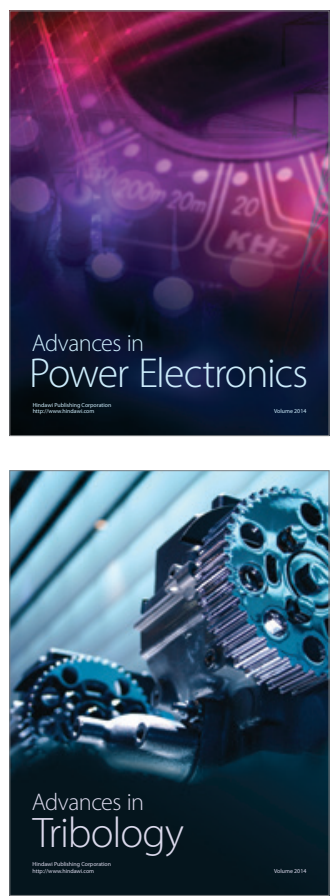

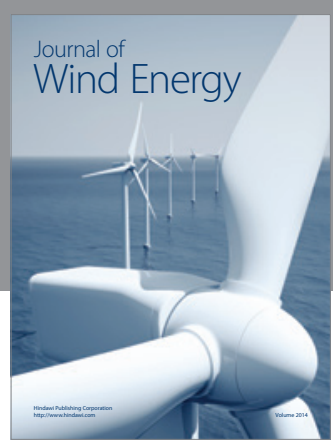

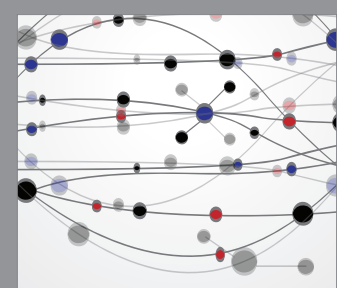

The Scientific World Journal

Submit your manuscripts at http://www.hindawi.com

Journal of

Structures
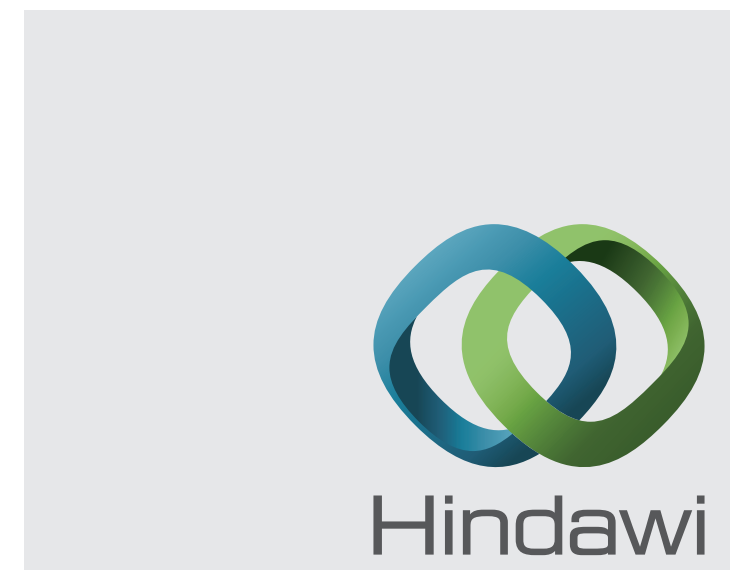

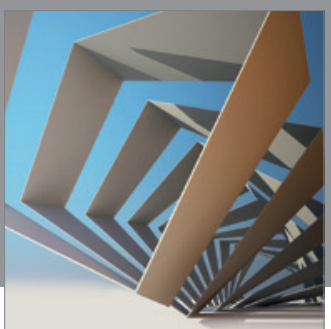

Rotating

Machinery
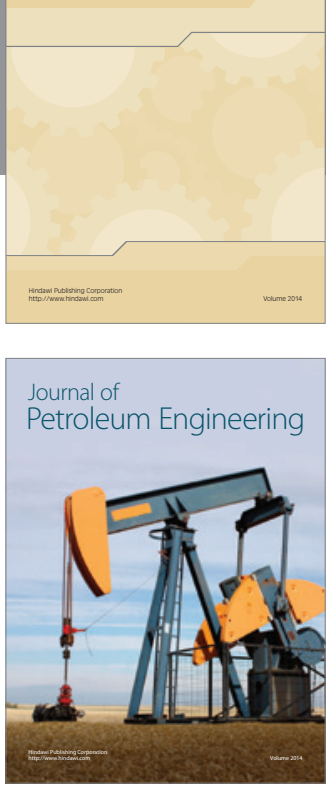

Journal of

Solar Energy
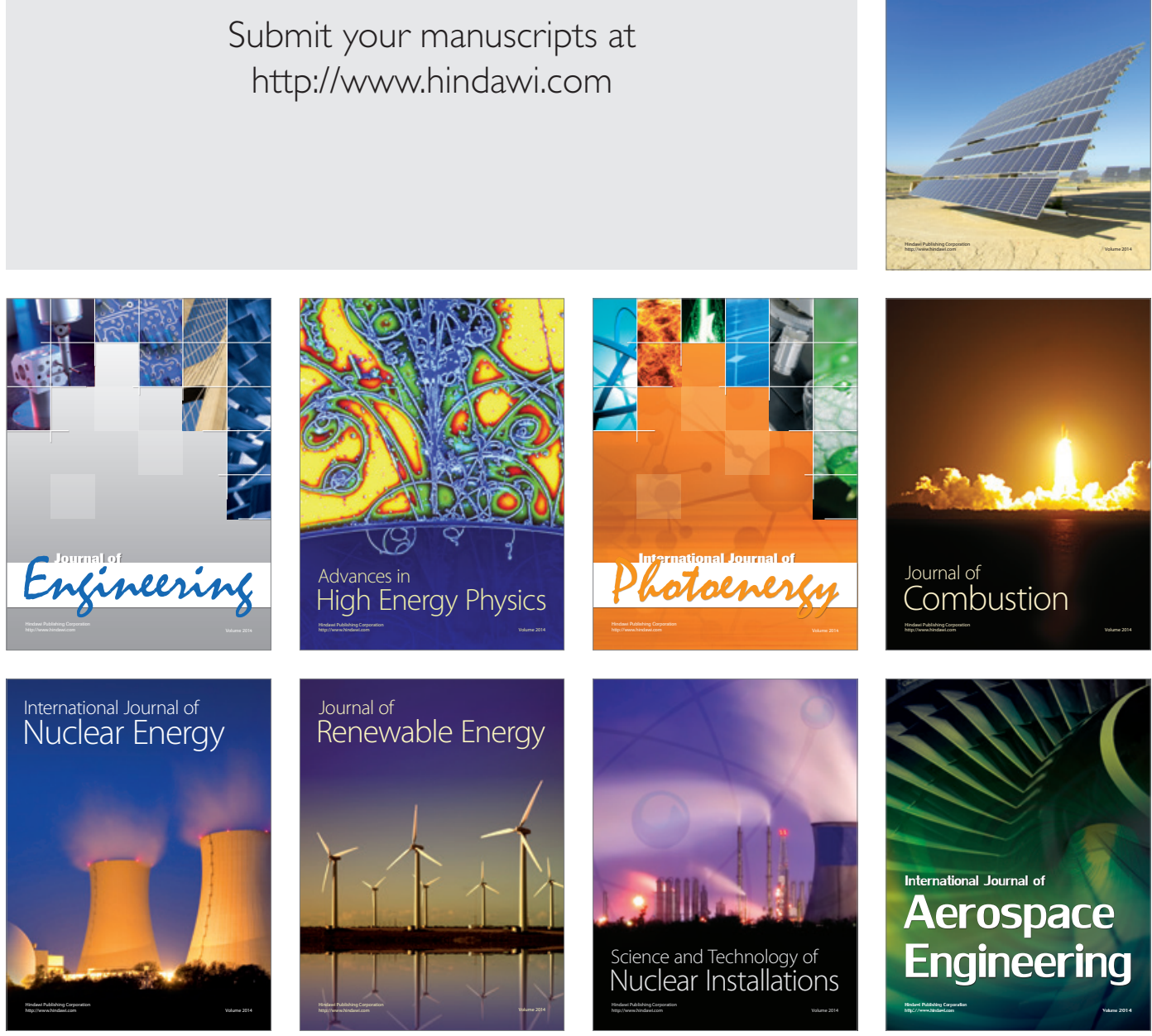